\title{
Flúor y fluorosis dental
}

\section{Fluoride and dental fluorosis}

Noelia Yesica Martínez Cántaro ${ }^{1 a}$

Yhamile Machaca Pereyra ${ }^{1 b}$

Luis Angel Cervantes Catacora ${ }^{1 b}$

Edison Ronaldo Mamani Torres ${ }^{1 b}$

Adriana Alejandra Laura ${ }^{1 b}$

Marcela Sofía Chambillo Nina ${ }^{16}$
iD https://orcid.org/0000-0003-0626-1773
iD https://orcid.org/0000-0002-1103-0868
iD https://orcid.org/0000-0001-7233-1835
iD https://orcid.org/0000-0003-1802-1291
(iD) https://orcid.org/0000-0001-9743-1410
iD https://orcid.org/0000-0001-5503-7407

Correspondencia: nmartinezc@unjbg.edu.pe

\section{Resumen}

El flúor se clasifica como mineral, pero no es hallado como tal en la naturaleza; los fluoruros los encontramos en el suelo, el agua, así como en las plantas, los animales, el aire. Su existencia es necesaria para mantener una salud bucal correcta. Durante la formación de los dientes aporta mejorando la apatita del esmalte dental, pero su exceso lleva a una alteración en distintos niveles, la cual se conoce como fluorosis dental, causando una lesión en la estructura dentaria, debilidad frente a la caries dental y afectando la estética de la sonrisa; por tanto, se lleva a cabo esta revisión con la finalidad de profundizar los conocimientos del accionar del flúor en el esmalte dental y el resultado frente a su inadecuada administración en nuestro organismo.

Palabras clave: flúor, fluorosis dental, esmalte dental, remineralización

\begin{abstract}
Fluoride is classified as a mineral, but it is not found as such in nature. Fluorides are found in the soil, water, plants, animals, and air. Its existence is necessary to maintain proper oral health. During the formation of the teeth it contributes improving the apatite of the dental enamel, but its excess leads to an alteration at different levels, which is known as dental fluorosis, causing an injury to the dental structure, weakness of the teeth and dental caries affecting the smile aesthetics, therefore, this review is carried out in order to deepen the knowledge of the action of fluoride in dental enamel and the result of its inadequate administration in our body.
\end{abstract}

Keywords: fluoride, dental fluorosis, tooth enamel, remineralization

\footnotetext{
${ }^{1}$ Universidad Nacional Jorge Basadre Grohmann. Facultad de Ciencias de la Salud. Escuela Profesional de Odontología. Semillero de Investigación e Innovación. Tacna, Perú

a Maestro en Odontología con mención en Patología

${ }^{\mathrm{b}}$ Estudiante
} 


\section{Introducción}

El flúor se encuentra en la naturaleza en un estado único de oxidación; forma enlaces de modo covalente e iónico. ${ }^{1}$ Por otro lado los fluoruros van a aumentar la resistencia del esmalte y a la vez reprimir el proceso carioso; ${ }^{2}$ tienen como acción evitar considerablemente la desmineralización y de igual manera estimular la remineralización del esmalte modificando la hidroxiapatita del esmalte en fluorapatita, siendo este más resistente a la desmineralización. ${ }^{3}$

Un exceso en la concentración de flúor afecta las conexiones de la célula matriz en dientes en formación, causando deficiencia en la maduración del esmalte y desencadenando una serie de porosidades en la superficie del mismo, ${ }^{4}$ manifestándose la fluorosis dental, que es considerada como una irregularidad dental, por tener una conducta epidemiológica con características endémicas, produciendo daño a niños como también a adolescentes, sin importar el lugar de origen. $^{5}$

La fluorosis dental implica todas las clases de fluoruro que se ingieren durante la fase de formación dental. La cantidad de fluoruro que contienen los alimentos, el agua, la sal fluorada, los enjuagues bucales, las pastas dentales, etc. son fuentes de fluoruros a las que, en la actualidad, estamos expuestos, y una exposición elevada, produciría esta anomalía. ${ }^{6,7}$

\section{Flúor}

El flúor es miembro de la familia de halógenos con número y peso atómico más bajos, su símbolo es $\mathrm{F}$ y su número atómico es $9 .{ }^{8}$ De forma natural se halla en el consumo de agua en una escala relativamente reducida de concentración de 1 a $1.50 \mathrm{mg} / \mathrm{l}$, el cual proporciona condiciones ideales para el correcto desarrollo de huesos y órganos dentarios. ${ }^{9}$ Las personas estamos expuestas a diferentes tipos de compuestos con flúor, sean naturales como artificiales. ${ }^{1}$

Los fluoruros aumentan la resistencia del esmalte e inhiben el proceso de caries; ${ }^{2}$ además evitan la desmineralización e impulsan la remineralización del esmalte dental. Tiene la propiedad de transformar la hidroxiapatita en fluorapatita, la cual es más resistente a la desmineralización. Algunos autores mencionan su poder antibacteriano frente a microorganismos como estreptococo mutans y los lactobacilos ${ }^{3}$ y otros autores refieren que no inhiben significativamente el crecimiento de bacterias de la placa; ${ }^{10}$ sin embargo, ello no resta importancia al uso en el tratamiento y la prevención de caries mediante dentífricos de concentraciones de 1000-1500 ppm, colutorios bucales y comprimidos con $1-2$ mg de fluoruro. ${ }^{11}$

La absorción del flúor se da de forma pasiva; cuando llega al estómago se une al hidrógeno y forma el ácido fluorhídrico, se eleva en plasma después de 10 a 30 minutos de la ingestión, luego es distribuido en todo el cuerpo y se almacena en los huesos y dientes. ${ }^{12}$ Se atribuye que la leche materna aporta el $75 \%$ de concentración de flúor el cual va a ser retenido rápidamente por las estructuras óseas y dentales del nuevo ser. ${ }^{13}$ En la saliva se encuentra el flúor de forma dependiente de la del plasma y su concentración no es igual en toda la cavidad bucal. ${ }^{14}$

Las investigaciones realizadas sostienen la utilidad del flúor como prevención de la caries dental; sin embargo, no descartan la posibilidad de fluorosis debido a la sobreexposición al flúor producida por contribución de otras fuentes, como alimentos y contaminación ambiental. ${ }^{15}$

\section{Mecanismo de acción del flúor en los dientes}

La distribución de flúor en el esmalte se dispone antes del brote de la dentición decidua en la cavidad oral, luego de ello se inicia una absorción lenta de flúor superficial con predisposición en regiones porosas y de caries; sin embargo, existe una reducción del mismo en piezas dentales con 
pérdida de esmalte superficial por efecto del desgaste, por ello se reconoce que la adición de flúor al esmalte se da en tres etapas:

Etapa 1. En el desarrollo del esmalte la mayor concentración de flúor se da cuando el contenido proteico es alto, por lo que el flúor parece vincularse con proteínas, mientras que en la maduración del esmalte el contenido de proteínas va disminuyendo al igual que la concentración de flúor; resultando una menor cantidad del flúor que se concentra y deposita nuevamente en el mineral de la superficie del esmalte. ${ }^{3}$

Etapa 2. Luego de la calcificación dentaria, las piezas dentarias pueden permanecer sin irrupción por un tiempo. La acción dinámica del líquido intersticial sobre la superficie del esmalte incorporará una concentración baja de flúor que, durante un periodo considerable, se acumulará cantidades sustanciales del mismo. ${ }^{3}$

Etapa 3. Después de la erupción, el flúor puede acumularse de manera pausada en el esmalte superficial desde el medio bucal. ${ }^{15}$

El fluoruro actúa si es libre soluble en el medio bucal (líquido de biopelícula o saliva). ${ }^{16}$ Su mecanismo de acción reside en el descenso de la desmineralización y la estimulación de remineralización del esmalte. ${ }^{17}$ Por lo que se ha encontrado que, aun a menor concentración, permanece en la desmineralización de la hidroxiapatita por su estado de sobresaturación con relación al esmalte, lo que facilitará su afluencia hacia los cristales, sustituyendo los hidroxilos por fluoruro, activando así el progreso de remineralización; de esta manera el fluoruro se concentrará en la superficie de los cristales parcialmente desmineralizados captando iones de calcio y creando fluoruro de calcio, que se establecerá formando en la superficie fluorhidroxiapatita, produciendo cristales más estables y resistentes. ${ }^{18}$

Inicialmente una lesión cariosa presenta una porosidad en el esmalte subsuperficial por efecto de periodos de desmineralización; clínicamente se percibe una opacidad denominándose "mancha blanca". Para ello la aplicación frecuente de dosis bajas de fluoruro de forma tópica favorece la remineralización más profunda en contraste a que si las dosis de fluoruros fuesen más elevadas. La alta concentración de flúor en dientes en formación produce retención de amelogeninas por la hipomineralización del esmalte, evidenciando un esmalte afectado con presencia de porosidades. $^{19,20}$

La acción antibacteriana del flúor por debajo de las 10 ppm no afecta el metabolismo bacteriano, pues si existe una lesión inicial de mancha blanca, el fluoruro no puede reponer el área porosa dentro del esmalte con minerales, pero sí ayudará a detener el progreso de la lesión; tendrá una superficie brillante como resultado del pulido y remineralización de la superficie en presencia de fluoruro, pero el aspecto blanco de las áreas porosas debajo, permanecerá parcialmente. ${ }^{21}$

\section{Vías principales de administración de flúor}

Vía sistémica: incluye el agua fluorada (contienen aproximadamente $0.7-1.2 \mathrm{mg}$ ), alimentos como pollo, lechugas, salmón ( 0.01 a $0.17 \mathrm{mg} / 100 \mathrm{~g}$ ) y el té (de 1 a $6 \mathrm{mg} / \mathrm{litro})$. Se recomienda en personas adultas; entre $3 \mathrm{mg} /$ día para mujeres y $4 \mathrm{mg} / \mathrm{d}$ ía para varones.

Vía tópica: pastas dentífricas, colutorios, geles y barnices. ${ }^{9}$

\section{Fluorosis dental}

La fluorosis dental es considerada como una anomalía que afecta a niños y adolescentes, debido a los altos contenidos de fluoruro en agua y alimentos ingeridos causando efectos endémicos. ${ }^{5}$ Durante la etapa de desarrollo de los dientes, cualquier tipo de digestión de fluoruro, de acuerdo a la severidad, afectará las estructuras dentarias. ${ }^{22}$ 
La fluorosis daña la estética de la sonrisa, se manifiesta con manchas blanquecinas, cafés, amarillas y, en una mayor severidad, causa la pérdida del esmalte dentario. Asimismo, provoca susceptibilidad a proceso carioso y pérdida de la estructura dentaria. ${ }^{7}$

\section{Características de la fluorosis dental}

La fluorosis dental se caracteriza por la alteración de los ameloblastos en el proceso de calcificación de la matriz durante el desarrollo de los dientes.

El proceso ocurre a nivel tisular cuando el flúor interactúa con los tejidos en la mineralización. El individuo estuvo expuesto a grandes cantidades de flúor durante mucho tiempo, lo que ocasionará manchas blancas en la corona, translucidez del esmalte, falta de brillo y opacidad del diente. ${ }^{23}$

Debido a que la fluorosis es una displasia del esmalte, esta dependerá del grado y tiempo que estuvo en contacto con el agente causal. ${ }^{3}$ En los casos más leves se presentan manchas blanquecinas y translucidez del esmalte sin dentina, prioritariamente se observan en el tercio medio e incisal o superficies oclusales. En los casos más graves aparecen manchas oscuras, cafés o amarillas, alterando la estructura dentaria con hipo calcificaciones.

Por ello, mayores cantidades a $1.5 \mathrm{mg} /$ /itro de ingesta de flúor durante la etapa del desarrollo dental serán las causantes de la fluorosis dental. ${ }^{24}$ Las manifestaciones clínicas estarán condicionadas a la cantidad de fluoruro ingerido, al tiempo de la exposición, la edad, el peso y el estado nutricional de cada individuo.

\section{Causas de la fluorosis dental}

La causa posible de la hipoplasia del esmalte es la demora en la hidrólisis y remoción de las proteínas del esmalte durante su desarrollo. El retardo es debido a la interacción del flúor con las proteínas en la etapa de desarrollo de la matriz. Los altos niveles de fluoruro que estarán disponibles retardarán la inhibición de los cristales de apatita, lo que afectará la maduración y calcificación del esmalte, formándose porosidades, además de obtener un esmalte muy débil. ${ }^{25}$

Al haber una cantidad de fluoruro libre en la matriz orgánica se impedirá la inhibición de los cristales de apatita de las enamelaminas que retrasarán la maduración del esmalte, ${ }^{26}$ si esta sobrepasa los 8 a 10 ppm no solo afectará la dentadura, sino también se presentará la fluorosis esquelética.

El grado de fluorosis dental va a estar directamente relacionado a tres aspectos: tiempo, duración y dosis. Clínicamente, su distribución se percibe de apariencia simétrica y horizontal estriada, aunque a todas las piezas no afecta de la misma manera, por lo que, a mayor tiempo de exposición a los fluoruros, tardará más en hacer erupción el diente y habrá una mayor gravedad de fluorosis.

Thylstrup \& Fejerskov propusieron una clasificación según los efectos biológicos del flúor denominado índice de TF, basados en los diferentes grados hepatológicos propios de la fluorosis dental y en los cambios adamantinos que se observan en la superficie dental en diez diferentes categorías de la fluorosis dental (Figura 1). ${ }^{27}$ 


\section{Figura 1}

Índice TF- Thylstup y Fejerskov

\begin{tabular}{l} 
Índice de Thylstup y Fejerskov (1978) \\
\hline TF0
\end{tabular} \begin{tabular}{l} 
Caracterizado por un esmalte normal, liso, traslúcido y cristalino, de color \\
uniforme. Estas características permanecen, aun después del secado con aire \\
prolongado. \\
Esmalte liso, traslúcido y cristalino con finas bandas horizontales de color \\
blanquecino. \\
\hline TF1
\end{tabular}

\section{Aplicación clínica en la fluorosis dental}

La fluorosis dental afecta principalmente la estética y, generalmente, se conoce a la microabrasión y el blanqueamiento externo como mejores resultados en pacientes con fluorosis dental leve.

La literatura describe diversos tipos de tratamiento para cada tipificación de fluorosis. En los casos de TF1 al TF3 se realizará un blanqueamiento y microabrasión. Para los casos de TF4 al TF6 se procederá a realizar la macroabrasión seguida de una microabrasión y blanqueamiento, considerándose necesarias también restauraciones. En el caso de TF7 se realizará restauraciones con resinas compuestas directas. Para TF8 y TF9, carillas y coronas completas. En la actualidad existen resinas llamadas infiltrantes, no será necesario realizar la cavidad debido al uso del ácido clorhídrico al $15 \%$ en lugar del ácido fosfórico, lo que permite la infiltración de la resina; sin embargo, es necesario un buen diagnóstico para seleccionar las indicaciones terapéuticas necesarias. ${ }^{28}$

El periodo de riesgo de cambios estéticos en los dientes permanentes es entre los 20 y los 30 meses de edad. El nivel recomendado para la ingesta diaria de flúor es de $0.07 \mathrm{mg} \mathrm{F} / \mathrm{Kg} /$ día (Tabla 1), lo que se considera de gran ayuda en la prevención de la caries dental, actuando en la remineralización. Una ingesta diaria superior a este nivel seguro aumenta el riesgo de fluorosis dental. Los procedimientos actualmente recomendados para el diagnóstico de fluorosis deben discriminar entre patrones simétricos y asimétricos y/o discretos de defectos opacos. La fluorosis se puede prevenir teniendo un conocimiento adecuado de las fuentes de fluoruro, sabiendo cómo manejar este problema y, por lo tanto, evitar la sobreexposición. ${ }^{29}$ 
Tabla 1

Nivel recomendado para la ingesta diaria de flúor

\begin{tabular}{ll}
\hline Infantes(meses) & $\mathrm{mg} / \mathrm{día}$ \\
\hline $0-6$ & 0.01 \\
$7-12$ & 0.5 \\
\hline
\end{tabular}

\begin{tabular}{ll}
\hline Infantes(meses) & $\mathrm{mg} / \mathrm{día}$ \\
\hline $0-6$ & 0.7 \\
$7-12$ & 0.9 \\
\hline
\end{tabular}

\begin{tabular}{ccc}
\hline edad & Hombres & Mujeres(mg/día) \\
\hline $1-3$ & 0.7 & 0.7 \\
$4-8$ & 1 & 1 \\
$9-13$ & 2 & 2 \\
$14-70$ & 4 & 3 \\
+70 & 4 & 3 \\
embarazo & & 3 \\
lactancia & & 3 \\
\hline
\end{tabular}

\begin{tabular}{ccc}
\hline edad & Hombres(mg/día) & Mujeres(mg/día) \\
\hline $1-3$ & 1.3 & 1.3 \\
$4-8$ & 2.2 & 2.2 \\
$9-13$ & 10 & 10 \\
$14-70$ & 10 & 10 \\
+70 & 10 & 10 \\
embarazo & & 10 \\
lactancia & & 10 \\
\hline
\end{tabular}

\section{A}

A. Ingesta adecuada recomendada según el Departamento de Nutrición del IOM (Institute of Medicine: Instituto de Medicina). B. Ingesta máxima tolerable de flúor para individuos sanos establecida por el Instituto de Medicina de la Academia Nacional de Ciencias (Institute of Medicine of the National Academy of Sciences).

\section{Discusión}

En las últimas décadas, la prevalencia de la fluorosis ha aumentado en todo el mundo con una cantidad porcentual que va de 7.7 al $80.7 \%$, en el que se cuenta con agua fluorada, y entre $2.9 \%$ al $42 \%$ en áreas sin este elemento. ${ }^{30}$

En países como Alemania, el 67 \% de la población consume sal fluorada; y en Suiza, el 85 \%. La fluorización de la sal se da en México, Colombia, Perú y Cuba, por lo que muchos países de América Latina han llegado a la cobertura del 90 al $99 \%$. $^{31}$

El consumo prolongado de agua con altos niveles de flúor (4 a $15 \mathrm{ppm}$ ) puede tener consecuencias más serias y provocar una fluorosis esquelética. Una encuesta realizada en el norte de Tanzania reveló que los sujetos de edad avanzada que, generalmente, bebían agua con un contenido de flúor superior al estándar tenían anomalías en los huesos. La fluorosis esquelética tiene la capacidad de causar dolencia dorsal y rigidez o deformidades neurológicas. ${ }^{22}$

Otra investigación determinó que el principal causante de esta situación clínica es el tragar la pasta durante el cepillado, además los padres de niños menores de 3 años en Dinamarca informaron que ocasionalmente el $12 \%$ de los niños comen pasta de dientes de un tubo. ${ }^{24}$ Los estudios en niños también indicaron que el periodo crítico de sobreexposición al flúor ocurre en los primeros tres años, y el mayor riesgo de fluorosis se da en el incisivo central superior permanente. ${ }^{32,33}$ También se encontró que el periodo crítico de los cambios permanentes de los dientes anteriores es entre los 15 y 24 meses, mientras que en las niñas se estima que sucede entre los 21 a 30 meses; comprobando, de esta manera, que existe una relación directa entre la cantidad de flúor ingerida durante el desarrollo de los dientes y la fluorosis dental. ${ }^{22}$ Estudios recientes en Perú determinan que solo el $53 \%$ de las pastas fluoradas contienen la concentración de fluoruro soluble total por encima de 1000 ppm, la cual es la concentración mínima necesaria para que ocurra un efecto anticaries. $^{34}$ 
Existen varios factores de riesgo influyentes en la aparición de la fluorosis dental, los más comunes de estos factores pueden ser el alto contenido de concentración de flúor en el agua, la sal, variados productos alimenticios como el arroz, maíz y plátano; el uso desmedido de agroquímicos fluorados en cosechas, la ingesta de dentífrico sin un control adecuado, etc. ${ }^{30}$

Cabe mencionar que el impacto de la presencia de fluorosis dental se relaciona con la calidad de vida en adolescentes. Constante $\mathrm{C}$ y colaboradores, en su investigación, mencionan también el factor socioeconómico, el cual restringe la oportunidad del acceso a servicios odontológicos, entendiéndose que a una condición socioeconómica baja predispone un mal estado de salud bucal. $^{35}$

Para mejorar la salud oral en niños se necesitan políticas en salud que promuevan e impulsen el acceso de servicios de salud oral de manera específica a los grupos de la población al que van dirigidos. En ese sentido, tener una información exacta de la distribución territorial de los problemas de salud otorgaría una mejor comprensión de las patologías e incrementaría la buena toma de decisiones. ${ }^{36}$

\section{Conclusiones}

El flúor es un elemento esencial para los seres humanos; controla la caries dental, da resistencia al esmalte, pero su exceso durante la fase formativa del desarrollo dental puede ser perjudicial para la salud, provocando una fluorosis dental, sobre todo en la población infantil.

La severidad de la fluorosis dental se relaciona directamente con la dosis, duración y tiempo de exposición al flúor, causando mayor susceptibilidad a la caries, afectando los cristales del esmalte con una apariencia de mancha blanca hasta café oscuro. En situaciones graves se da la pérdida del esmalte y se generan pequeños hoyuelos cuando los elevados niveles de fluroruro se dan en la matriz orgánica, estos prohíben la división de las enamelinas con los cristales de apatita, aplazando la maduración de los cristales del esmalte, afectando su calcificación y forjando uno con alto contenido de flúor y porosidades; es decir, un esmalte endeble.

Se requiere programas y políticas de salud para promover actividades de enfoque preventivopromocional que incluyan procesos educativos dirigidos a sensibilizar y capacitar a la población sobre el manejo idóneo de fluoruros tópicos y evitar la sobreexposición.

\section{Referencias}

1. Alfredo Rigalli Rodolfo C.P. Determinación de la concentración de flúor en muestras biológicas. Actualizaciones en Osteología. 2007;3(1):27-34.

2. Sosa Rosales MdIC. Evolución de la fluoruración como medida para prevenir la caries dental. Revista Cubana de Salud Pública. 2003;29:268-74.

3. J. GR. Fluorosis dental: metabolismo, distribución y absorción del fluoruro. [Internet]. . Revista ADM 2005 [citado 07 feb 2019];.LXII(6):225-9. Disponible en: http://www.medigraphic.com/pdfs/adm/od-2005/od056d.pdf.

4. Calderón Betancourt J, López Larquin N, Dobarganes Coca AM. Características generales de la fluorosis dental. Rev. electron. Zoilo [Internet]. 2014 [citado 7 Abr 2021];, 39(12):[aprox. 0 p.]. Disponible en: http://revzoilomarinello.sld.cu/index.php/zmv/article/view/128/223

5. Córdova D. Fluorosis dental en niños de 13 a 15 años del colegio Felipe Santiago Salaverry de Picsi. Chiclayo, Perú, 2009. Revistakiru 5(2): 72-77.

6. Alvarez JA, Rezende KM, Marocho SM, Alves FB, Celiberti P, Ciamponi AL. Dental fluorosis: Exposure, prevention and management. Med Oral Patol Oral Cir Bucal. 2009; 14(2): 103-107. https://www.ncbi.nlm.nih.gov/pubmed/19179949 
7. Bordoni N ERA, Castillo Mercado R. Odontología Pediátrica. La salud bucal del niño y el adolescente en el mundo actual. 1a. Buenos Aires: Médica Panamericana S.A; 2010.1142 p.

8. Gómez G GD, Martín M. . Flúor y fluorosis dental. 1ra ed. Canarias: Editorial Abiss $1^{\text {a }}$ edición: Santa Cruz de Tenerife 2002.

9. R. G. Fluorosis dental en estudiantes de 8 a 12 años de la escuela fiscal mixta Luis Vivero Espinoza de la parroquia Totoras en la ciudad de Ambato año lectivo 2010-2011. [Tesis doctoral]. Quito: UCE; 2011.

10. Rölla G, Ogaard B, Cruz RD. Topical application of fluorides on teeth. New concepts of mechanism of interaction. J Clin Periodontol 1993;20(2):105-8.

11. Sthephen KW. Fluoride Toothpastes, Rinses, and Tablets. Advances in Dental Research. 1994;8(2):185-9.

12. Ekstrand J, Whitford GM. Fluoride Metabolism. En: Ekstrand J, Fejerskov O, Silverstone L.editors. Fluoride in Dentistry. Copenhage: Munksgaard; 1988.

13. Gómez- Soler S. Fluorterapia en odontología. Fundamentos y Aplicaciones clínicas. $4^{\mathrm{a}}$ ed. Santiago de Chile, 2010:37-38.

14. Wlatherell JA, Robinson C, Ralph JP, Best JS. Caries Res 1984; 18: 343-53.

15. Williams R EJ. Bioquímica dental básica y aplicada. Cap. 15 y 16, Edit. Manual Moderno, México, 1989: 350.

16. Actas de un Simposio Internacional Conjunto IADR / ORCA sobre Fluoruros: Mecanismos de acción y recomendaciones de uso -dmd, Callaway Gardens Conference Center, Pine Mountain, Georgia. J Dent Res. Febrero de 1990; 69: Número especial.

17. Ellwood R FO, Cury JA, Clarkson B. . Fluorides in caries control. En: Fejerskov O, Kidds E, editors. Dental caries: The disease and its clinical management. Oxford: Blackwell; 2008.

18. Buzalaf M P, J, Honório H, ten Cate J. . Mechanisms of action of fluoride for caries control. Monogr Oral Sci. 2011 Jun; 22: 97-114.

19. Clemencia M. Vargas DDS P. Fluoride Supplements Prevent Caries but can Cause Mild to Moderate Fluorosis, . Journal of Evidence Based Dental Practice, USA, . 2011;11(1).

20. Al-Khateeb S ER, Angmar-Mänson B. Effect of acid-etching on remineralization of enamel white spot lesions. Acta Odontol Scand 2000;58:31-6. Acta Odontol Scand 2000;58(1):31-6.

21. Cury JA TL. Remineralización del esmalte: ¿controlar la enfermedad de caries o tratar las lesiones de caries tempranas? Braz Oral Res. 2009; 23 Supl. 1:23-30.

22. Hidalgo-Gato Fuentes I, Duque de Estrada Riverón J, Mayor Hernández F, Zamora Díaz JD. Fluorosis dental: no solo un problema estético. Revista Cubana de Estomatología. 2007;44:0-

23. Martín-González J.* SDB*TDML*CCL*LCJM*LFFJ*ySEJJ*. scielo. [Online].; 2015 [cited $2012 \quad \mathrm{novi}$ e m b e 6 . A v a i l a ble f r o m : http://scielo.isciii.es/scielo.php?script=sci_arttext\&pid=S0213-12852012000600004.

24. Mattos-Vela Manuel Antonio, Carrasco-Loyola Milagros Bertha, Valdivia-Pacheco Suelen Giuliana. Prácticas sobre uso de pasta dental fluorada en preescolares, padres y profesores. Odontoestomatología [Internet]. 2012 Nov [citado 2021 Abr 09] ; 14( 20 ): 38-48. Disponible en: http://www.scielo.edu.uy/scielo.php?script=sci_arttext\&pid=S168893392012000200005\&lng=es.

25. Espinosa Fernández R. VHR, Ceja Andrade I. Fluorosis dental. Etiología, diagnóstico y tratamiento. Editorial Ripano $1^{\text {a }}$ Edicion 2011.

26. Cardozo ML, Lucas, G. Q. Estudio de la Prevalencia y Severidad de Fluorosis Dental en Escolares Informe CyT Cátedra de Odontopediatría - Facultad de Odontología - UNNE. 2013.

27. A. IM. El fluoruro y los diferentes vehículos para prevenir la caries dental. Salud Dental para Todos [citado 16 Feb 2021];11: [12 pantallas]. Disponible en URL: http://www.sdpt.net/CAR/fluoruros.htm\#Gele. 
28. Cedillo Valencia J.J. CFJE. Resinas Infiltrantes, una novedosa opción para las lesiones de caries no cavitadas en esmalte. Revista ADM /VOL.LXIX. N¹, 2012: 38-45.

29. Abanto Alvarez J RK, Marocho SM, Alves FB, Celiberti P. , Ciamponi AL. Dental fluorosis: exposure, prevention and management. Med Oral Patol Oral Cir Bucal. 2009;14(2):E103E107. Published 2009 Feb 1.

30. Posada-Jaramillo GA, Restrepo-Puerta AM. Factores de riesgo ambientales y alimentarios para la fluorosis dental Andes, Antioquia 2015. Rev. Fac. Nac. Salud Pública. 2017; 35(1): 7990. DOI:10.17533/udea.rfnsp.v35n1a09

31. Marthaler TM. Salt fluoridation and oral health. Acta Med Acab. 2013; 42(2): 140-55. https://www.ncbi.nlm.nih.gov/pubmed/24308394

32. A. B. "Risk periods" associated with the development of dental fluorosis in maxillary permanent central incisors: a meta-analysis. Acta Odontol Scand. 1999; 57(5):247-56.

33. Evans WRS, W.J. 1999. . An epidemiologic estimate of the critical period during which human maxillary central incisors are most susceptible to fluorosis. Journal of Public Health Dentistry 51: 251 - 259 .

34. Acosta de Camargo, M. G., Palencia, L., Santaella, J., \& Suárez,. El uso de fluoruros en niños menores de 5 años. Revista de Odontopediatría Latinoamericana,2020; 10(1), 82-92.

35. Constante Cruz Adrián Paulo, Pérez Rosero Eliana Rosalía, Rodríguez Villarreal Juan Pablo, Cabrera Arias María Alejandra, Armas Vega Ana del Carmen. Impacto de la fluorosis dental en la calidad de vida de adolescentes ecuatorianos de entre 11 a 14 años. Odontología Vital [Internet]. 2020 June [cited 2020 Dic 14] ; ( 32 ): 21-28. Available from: http://www.scielo.sa.cr/scielo.php?script=sci_arttext\&pid=S1659$07752020000100021 \&$ lng=en.

36. Delamater PL MJ, Shortridge AM, Grady, SC. Measuring geographic access to health care: raster and network-based methods. InternationalJournal of Health Geographics. 2012;11(1):15. 